\title{
Safe Retinol-Like Skin Biological Effect by a New Complex, Enriched with Retinol Precursors
}

\author{
Ze'evi Ma'or',2, Dror Cohen',2, Ya'ara La'or-Costa, ${ }^{1,2}$, Meital Portugal-Cohen ${ }^{1,2}$ \\ ${ }^{1}$ Ahava Dead Sea Laboratories, Lod, Israel \\ ${ }^{2}$ The Skin Research Institute, the Dead-Sea and Arava Science Center, Masada, Israel \\ Email:meital.p@ahava.com, zeevi@ahava.com
}

How to cite this paper: Ma'or, Z., Cohen, D., La'or-Costa, Y. and Portugal-Cohen, M. (2020) Safe Retinol-Like Skin Biological Effect by a New Complex, Enriched with Retinol Precursors. Journal of Cosmetics, Dermatological Sciences and Applications, 10, 59-75. https://doi.org/10.4236/jcdsa.2020.102007

Received: April 19, 2020

Accepted: May 28, 2020

Published: June 1, 2020

Copyright $\odot 2020$ by author(s) and Scientific Research Publishing Inc. This work is licensed under the Creative Commons Attribution International License (CC BY 4.0).

http://creativecommons.org/licenses/by/4.0/

\begin{abstract}
Background: Retinol (RE) is deeply involved in skin processes, therefore it is widely formulated in cosmetics, primarily as an anti-aging ingredient. Despite its efficacy, the safety profile of RE is controversial. Objectives: Pretinol (PRE) complex was formulated with two RE precursors, $\beta$-Carotene and Niacinamide, in order to deliver retinol-like skin benefits with healthier characteristics, assuming that skin enzymes will enable safe RE supply on spot. Methods: The expression levels of hyaluronic acid, Tumor Necrosis Factor alpha (TNF $\alpha$ ) and Interleukin 1 alpha (IL-1 $\alpha$ ), were measured using various skin models before and after exposure to PRE and RE. Full genome microarray was performed and the affected genes and pathways were analyzed. Results: Following fibroblasts exposure to PRE, the natural synthesis of hyaluronic acid is significantly elevated. Skin safety, demonstrated via cytokines expression on ex-vivo skin, results with TNF $\alpha$ and IL- $1 \alpha$ elevation by RE application. In contrary PRE significantly reduces TNF $\alpha$ while IL- $1 \alpha$ is not affected. These results establish skin safety advantage of PRE vs RE. Microarray results examined on skin equivalents reveal the involvement of PRE in inflammatory attenuation. Conclusions: Formulating RE precursors as a safe source for RE is partially supported. PRE presents a skin benefit in parallel to RE, while PRE characteristics are suggested to be safer to skin.
\end{abstract}

\section{Keywords}

Retinol, Hyaluronic Acid, $\beta$-Carotene, Skin, Inflammaging, Irritation

\section{Introduction}

Vitamin A, the most multifunctional vitamin in the human body, constitutes a 
group of organic lipid-soluble compounds comprising retinol (RE) and its derivatives, mainly the RE esters, retinyl palmitate and retinyl acetate [1] [2] [3]. $\mathrm{RE}$ is deeply involved in growth and maintenance due to its cellular contribution to cell proliferation and differentiation from early embryogenesis to adulthood [4]. RE participates in many skin biological processes in epidermis and dermis and hence is formulated in a lot of cosmetic preparations, mainly as anti-aging and skin brightening agent [5] [6]. Its contribution to alleviating acne symptoms was proven and as a result it is widely formulated in dermo-cosmetic products aiming to treat acne-prone and oily to problematic skin conditions [7] [8]. Despite its proven efficacy, the safety assessment of RE is still controversial [9]. According to some reports, the topical applications of RE containing skin preparations might trigger severe side effects, such as dryness and irritation, skin color changes and sensitivity to sunlight [10]. This fragile safety status affects RE acceptance in cosmetics. Chemists all over the world are searching for new ways to develop safe RE cosmetics, i.e. formulating skin preparations with proven RE skin benefits, that are free off RE related side effects. Sophisticated delivery systems for RE, such as liposomes, nano and micro encapsulations were proposed and clinically tested [11], but these technologies did not solve the fragile status related to RE containing formulae. In some cases, the situation was even worsened, especially if enhanced RE percutaneous penetration was measured [12]. In order to achieve a desired safe retinol-like skin activity, the idea to formulate its precursors was raised, assuming that the natural skin enzymatic activity will effectively convert these precursors, and will enable the skin tissue a safe level of RE supply "on the spot". $\beta$-Carotene was chosen to serve as a successful RE precursor and a bio-available RE source [13], as it is a safe compound, that when processed by epidermal enzymes, is converted into retinyl esters to supply skin with RE [14]. Combined with Niacinamide, known as Niacin and Vitamin B3, as it is also involved in the final stage of the skin's natural process of retinol biosynthesis, mediated by RE dehydrogenase enzyme with Nicotinamide adenine dinucleotide Coenzyme A (NAD Co-A) [15] [16]. The natural skin process of RE biosynthesis from $\beta$-Carotene and Niacinamide as precursors is described in Figure 1 below.

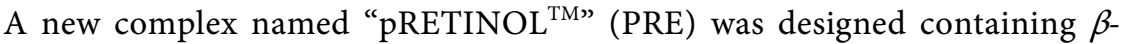
carotene and Niacinamide as RE precursors, with the addition of two Dead Sea natural actives, Dead Sea mineral rich water and an extract of Dunaliella Salina, Dead Sea alga. Dead Sea water skin benefits are well established and are widely formulated in cosmetics, mainly as a moisturizing humectant and an anti-aging active ingredient [17] [18]. Dunaliella Salina, a key micro-flora microorganism of The Dead Sea, was first discovered by Volcani in the Dead Sea saturated saline water in 1940 [19]. Following Dunaliella Salina exposure to sun the alga accumulates large quantities of $\beta$-carotene at its cell periphery [20]. The $\beta$-carotene accumulated level depends on water salinity, temperature and light intensity and could accomplish up to $10 \%$ of alga dry weight [19]. 


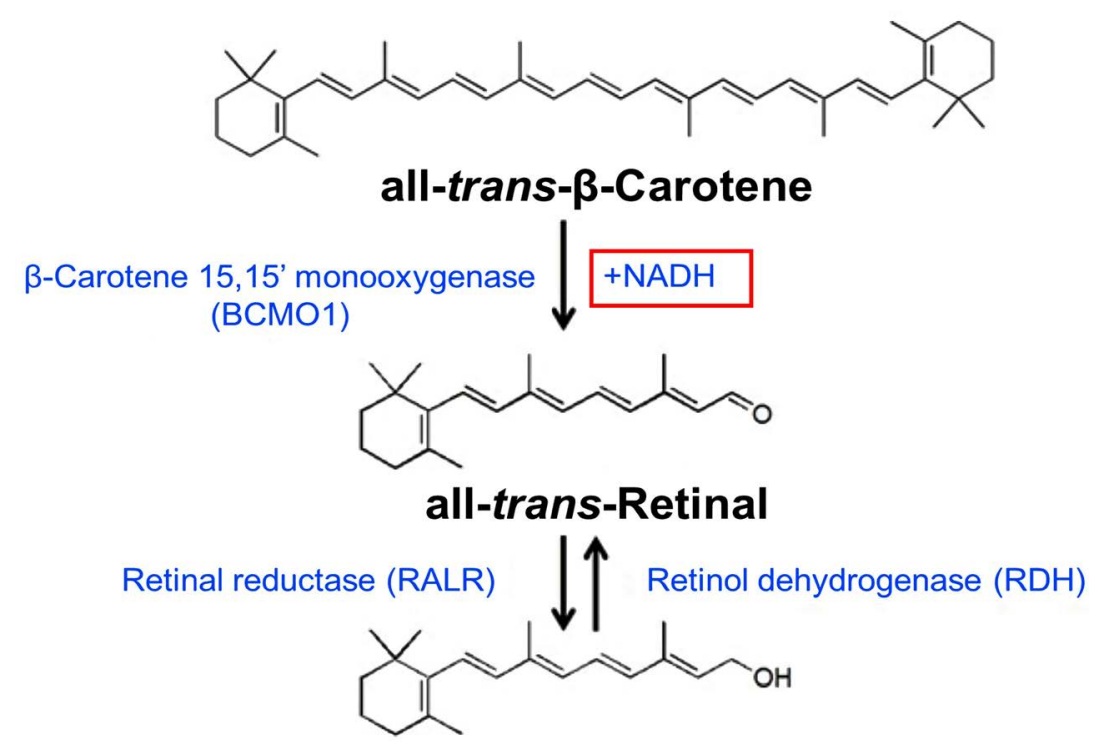

all-trans-Retinol

Figure 1. Retinol biosynthesis [2].

The Dunaliella Salina natural synthesis of $\beta$-carotene is believed to assist survival in the extreme Dead Sea conditions, especially when the exposure to harmful sun irradiation is high [21]. The bioactivities and skin safety of PRE complex, comprised of Dead Sea water, Dunaliella Salina alga, and RE precursors: $\beta$-carotene and Niacinamide, were elucidated. The expression levels of relevant biomarkers were measured using various skin models: In vitro Human dermal fibroblasts cell cultures, reconstructed 3D skin equivalent and ex-vivo human skin organ culture. In this work we present our test results and the new complex capabilities to introduce safe RE cosmetics.

\section{Methods}

\subsection{Preparation of Test Materials}

\subsection{1. pRETINOL-A Complex Enriched with Retinol Precursors}

pRETINOL (PRE) was prepared as a complex of 4 different active materials added and mixed together via vortex, including concentrated $\beta$-Carotene ( $\beta$-Carotene $10 \% \mathrm{CWS} / \mathrm{S}$ dispersed in a starch coated matrix. DSM), Niacinamide/pyridine3-carboxamide (KOYOWA HAKKO Europe GmbH), Dunaliella Salina commercial aqueous extract (PHEPA ${ }^{\circledR}$-CTIVE, DSM), and Dead Sea water extract (Osmoter $^{\mathrm{TM}}$ by AHAVA-Dead Sea Laboratories Ltd.) collected from the Dead Sea lake as describe at Portugal-Cohen et al. [17]. The relative ration of four PRE complex compounds is $0.0156 \beta$-Carotene: 1 Niacinamide: 30 Dunaliella Salina aqueous extract: and 5 Dead Sea water extract (w/w/v/v). PRE was diluted before application with distilled water according to test protocols. For HSOC skin test, PRE complex was diluted with distilled water before skin application to a final concentration of $3.6 \% \mathrm{w} / \mathrm{w}$. On fibroblasts cell culture test, PRE complex was added to fibroblast cell culture media to a final concentration of $1.44 \%(\mathrm{v} / \mathrm{v})$. 


\subsubsection{Retinol}

Retinol (RE) (BASF, Retinol 50C), contains $46.6 \%$ all-trans retinol, $49 \%$ polysorbate $20,1.0 \%$ of Butylhydroxyanisole, and $3.4 \%$ of Butylhydroxytoluene. RE was diluted with ethanol according to test protocols. On HSOC skin test, $3 \mu \mathrm{l}$ of $1 \%(\mathrm{w} / \mathrm{w}) \mathrm{RE}$ in ethanol, was topically applied. On dermal Fibroblasts test, 1.0 $\mu \mathrm{M}$ of $\mathrm{RE}$ were added to fibroblast culture medium.

\subsection{Ex-Vivo Human Skin Organ Culture Preparation Protocols}

EX-vivo human skin organ culture (HSOC) was prepared as described in Portugal et al. [22]. Human skin cultures were obtained from healthy females, aged 37 - 60, undergoing abdominal plastic surgery in accordance with Hospital Ethics Committee approval. $0.64 \mathrm{~cm}^{2}$ fixed-size of explant skin pieces were cut from donated skin tissue, placed with dermal side down in culture medium Dulbecco's Modified Eagle's High D glucose medium (DMEM), supplemented with 100 $\mathrm{U} / \mathrm{ml}$ penicillin and $100 \mu \mathrm{g} / \mathrm{ml}$ streptomycin), and incubated overnight at $37^{\circ} \mathrm{C}$ with $5 \% \mathrm{CO}_{2}$ for $24 \mathrm{~h}$ of recovery. Following incubation ex-vivo HSOC pieces were treated topically with tested materials.

\subsection{Human Primary Fibroblast Cell Culture Protocols}

In-vitro human primary dermal fibroblast cell culture was obtained based on the protocol described by Limat et al. [23]. Human primary fibroblast cells were grown in cultures to $80 \%$ confluency with DMEM culture medium, enriched with $10 \%$ fetal bovine serum $100 \mathrm{U} / \mathrm{ml}$ penicillin, and $100 \mathrm{U} / \mathrm{ml}$ streptomycin. The cells were cultivated in 6 wells plates. Tested materials were diluted before application as follows: PRE complex was diluted $\times 2.5$ with culture medium DMEM. RE was added to the culture medium at a final concentration of $1.0 \mu \mathrm{M}$. Fibroblast cells were incubated at $37^{\circ} \mathrm{C}, 5 \% \mathrm{CO}_{2}$ for $48 \mathrm{~h}$. After incubation, the cells were extracted using RIPA Lysis Buffer System (Santa Cruz Biotechnology) collected to a blending Eppendorf and ground using stain steal beads and a bullet blender tissue homogenizer (Bullet blender blue, Next Advance) at maximum speed for $5 \mathrm{~min}$. The samples were centrifuged at $4^{\circ} \mathrm{C}$ for $15 \mathrm{~min}$ at $5000 \mathrm{rpm}$, and the supernatant was collected. Protein content on epidermis lysate was determined using BCA Protein assay [24]. The protein level for all samples was diluted with PBS to reach a final concentration of $0.1 \mathrm{ng} / \mathrm{ml}$. Samples were tested for Hyaluronic Acid (HA) using ELISA assay kit (R\&D Systems). Results are presented as $\mathrm{ng} / \mathrm{ml}$ of HA per $1.0 \mu \mathrm{g}$ of protein.

\subsection{Reconstructed Skin Model}

Full Thickness reconstructed skin tissues (MatTek EFT-400 full-thickness skin tissues) was used as described in Portugal et al. [25]. Skin pieces were placed into a 6-well plate containing $2.5 \mathrm{ml}$ of assay medium and incubated overnight at $37^{\circ} \mathrm{C}$ and $5 \% \mathrm{CO}_{2}$. Then, the assay medium was replaced with $5 \mathrm{ml}$ of fresh medium and the tissues were treated topically with tested materials for $48 \mathrm{~h}$. At the end of 
the incubation period the surfaces of the tissues were rinsed with PBS to remove the test materials, the tissues were homogenized for RNA/protein's isolation, and the tissue culture media was collected for ELISA methods.

\subsection{RNA Isolation Protocol}

Total RNA was extracted from $48 \mathrm{~h}$ reconstructed skin using RNAqueous kit (Ambion) followed by mRNA Amplification protocol (Ambion, MessageAmp aRNA kit). Total RNA was reverse-transcribed to cDNA using a T7 oligo (dT) primer. Second-strand cDNA was synthesized and in vitro transcribed to anneal RNA (aRNA). The RNA concentration was determined by Ribogreen assay reagent (Molecular Probes) using Thermo Labsystems Fluorskan Ascent FL fluorometer after assessing its RNA quality via gel electrophoresis.

\subsection{Human Gene Expression/Affymetrix Microarray Protocol}

The expression of human genes was analyzed as described in literature [25]. aRNA probes from skin samples, treated with test materials (i.e. PRE complex and RE) were labeled with Cy3 and Cy5 (PerkinElmer, ASAP RNA Labeling Kit) and Purified by Millipore Microcon YM-30 filter column and TE buffer (Invitrogen). After purification, the fluorescent aRNA probes prepared above were hybridized to Agilent DNA Microarray Chip for $17 \mathrm{~h}$ at $65^{\circ} \mathrm{C}$. The microarrays signal was scanned (Axon GenePix, 4100A Scanner) with the scanning resolution set to $5 \mu \mathrm{m}$ and analyzed with GenePix Pro software. During the initial scan the photomultiplier tube (PMT) gains for the scanner were adjusted such that the cy5/cy3 image count ratios were between 0.95 and 1.05. Fluorescence intensities for the microarrays were subjected to global normalization by quantile method and were expressed as adjusted logFCs.

\subsection{Gene Set Enrichment Analysis (GSEA)}

The GSEA software was downloaded from the Broad Institute [26]. The adjusted logFCs were used for ranking the whole transcriptome and GSEA (cut-off independent) was carried out as described previously (Subramanian et al., PNAS 2005) using the MSigDB v6.1 database category $\mathrm{H}$

(http://software.broadinstitute.org/gsea/msigdb/index.jsp). Enrichment of gene sets was considered statistically significant if the false discovery rate (FDR) was $<0.05$.

\subsection{Hyaluronic Acid Quantification Test Protocol}

The expression level of Hyaluronic Acid (HA) was assessed via ELISA test performed as described by Portugal et al. [25]. In brief, $0.1 \mu \mathrm{g} / \mathrm{ml}$ of hyaluronic acid diluted in PBS was applied to human primary fibroblast culture. The lysate was transferred to a 96 wells plate that was previously coated with Hyaluronic acid coating antibodies and blocked with BSA. After 2 hours of incubation at RT the wells were washed with PBS solution contains $0.5 \%$ of Tween20 (Sigma) (TBPS). 
Hyaluronic acid detection antibody solution was transferred to each well and the samples were incubated at RT for $2 \mathrm{~h}$ and washed with TPBS wash buffer. A Streptavidin-HRP solution then added and incubated for $20 \mathrm{~min}$ at RT and washed thoroughly with TPBS wash buffer. TMB substrate solution was added and put at RT for $20 \mathrm{~min}$. The reaction was stopped with stop solution and the plate was read at $570 \mathrm{~nm}$ and $450 \mathrm{~nm}$ for correction.

\subsection{TNF $\alpha$ and IL-1 $\alpha$ Cytokines Quantification Test Protocols}

The expression level of Tumor Necrosis Factor alpha (TNF $\alpha$ ) and Interleukin 1 alpha (IL-1 $\alpha$ ) Cytokines on skin culture media were quantified using specific ELISA kits for TNF $\alpha$ and IL-1 $\alpha$ (Biolegend) as describe by Portugal et al. [25]. HSOC skin pieces were topically applied with tested materials, PRE complex or $\mathrm{RE} 1 \%$ in ethanol, and incubated at $37^{\circ} \mathrm{C}$, under $5 \% \mathrm{CO}_{2}$ for $48 \mathrm{~h}$. After $48 \mathrm{~h}$ incubation, the culture media were collected and transferred to ELISA assay to measure the quantity of the cytokine biomarkers, TNF $\alpha$ and IL- $1 \alpha$ (Biolegend).

\subsection{UVB Induced Cytokine IL-1 $\alpha$ and TNF $\alpha$ Expression Test Protocol}

UVB induced skin inflammation level was evaluated using ELISA assays kit for the cytokine IL-1 $\alpha$ expression, as described in Meital et al. [22]. HSOC pieces were topically applied with $3 \mu \mathrm{l}$ of test materials. After $24 \mathrm{~h}$ of incubation at $37^{\circ} \mathrm{C}$ $5 \% \mathrm{CO}_{2}$ HSOC pieces were irradiated with UVB lamp at a total energy of 200 $\mathrm{mJ} / \mathrm{cm}^{2}$ and skin was topically applied again, immediately after irradiation, with two test materials, 3.6\% (w/w) PRE complex and RE in a final concentration of $1 \% \mathrm{w} / \mathrm{w}$. HSOC pieces were incubated for $24 \mathrm{~h}$ at $37^{\circ} \mathrm{C}$, under $5 \% \mathrm{CO}_{2}$ for $24 \mathrm{~h}$. After $24 \mathrm{~h}$ incubation, the media was collected and transferred to ELISA assay to measure the expression level of the cytokines IL-1 $\alpha$ (Biolegend).

\subsection{Additional Statistical Tools}

All data are expressed as the mean \pm standard error (SE) of at least in triplicate and for three different experimental occasions. A statistic student's T-test was performed for each independent average value. Differences were considered significant at $\mathrm{p}<0.05$.

\section{Results}

\subsection{Genes and Biological Pathways Affected by Skin Exposure to PRE}

Skin bioactivity following its exposure to PRE vs RE was investigated. As a first step, full human gene transcription was studied. Skin equivalent samples were applied with PRE. The effects of these treatments on various biological functional gene sets were analyzed by the GSEA approach. The changes in expressed gene level following PRE treatment were evaluated using human genome microarray, as described in the Methods part. A group of major skin related pathways 
was selected from KEGG and Wikipathways lists. The significant skin biological pathways, affected by PRE application, and their calculated p-value, False Discovery Rate (FDR) and z-score are presented in Table 1.

\subsection{Gene Profile Following Skin Exposure to PRE vs RE}

Each array was processed and normalized the data from all arrays to get the average expression and $\log$ fold change $(\log \mathrm{FC})$ for each gene on each array $(19,245$ evaluated genes in total). From the heatmap, where the individual values contained in a matrix are represented as colors, it can be seen that PRE shows a different pattern from RE. Yet, the up-regulated genes in the middle of the graph seem to be common among the two groups. The heatmap of the affected biological pathways to PRE, vs RE is presented in Figure 2.

Comparison of the gene profiles of the treatment groups PRE and RE to whole transcriptome GSEA revealed several biological pathways relevant to skin inflammation (no. 1, 2, 3, 4), apoptosis (no. 5) and DNA base repair (no. 6) as shown in Table 2. A summarizing table with $\mathrm{Z}$ scores results of the two treatments is presented in Table 2.

Comparison of the results suggests that PRE reduces inflammation and apoptosis related pathways versus $\mathrm{RE}$ as indicated by $\mathrm{Z}$ score. Moreover, it increases DNA repair pathway unlike RE.

\subsection{Biological Efficacy and Safety Effects Following Skin Exposure to PRE vs RE}

The biological effect of PRE on skin was further tested compared to RE in terms of safety and activity. In vitro Human dermal fibroblasts cell cultures and ex-vivo HSOC were employed to measure the expression levels of standard biomarkers. $\mathrm{HA}$, a polysaccharide involved in skin hydration maintenance, was selected as a positive dermal RE biomarker due to its known upregulation, following skin exposure to RE [27]. The test samples were compared to untreated dermal fibroblast cells. The results are presented in Figure 3.

The results show significant HA elevation of $70 \%$ following PRE treatment that was similar to RE treatment, increased the HA by $60 \%$ in comparison to untreated fibroblast cells.

For assessing PRE skin safety, the levels of expressed cytokine biomarkers, TNF $\alpha$ and IL- $1 \alpha$ were studied. The expressed level of TNF $\alpha$, a common skin inflammation biomarker, and the level of IL- $1 \alpha$, a common skin irritation bio-marker. These results are presented in Figure 4.

The results show significant TNF $\alpha$ elevation of $102 \%$ and IL- $1 \alpha$ of $112 \%$ following RE treatment, while PRE treatment significant reduced the levels of TNF $\alpha$ by $50 \%$ and IL- $1 \alpha$ level is not affected. In the next step the two cytokines, TNF $\alpha$ and IL- $1 \alpha$, were tested on UVB irradiated HSOC. This model imitates skin following sun exposure and allows to predict PRE safety on sun-exposed skin. The results are presented in Figure 5. 
Table 1. The biologic pathways names, number of affected genes, calculated $\mathrm{p}$ value, FDR and Z-score of gene expression of skin equivalents following PRE application. Positive Z-score value indicates that the pathway tends to be up-regulated, a negative $\mathrm{Z}$-score means the pathway tends to be down-regulated. Significance is higher as absolute value of Z-score is bigger, as well as $\mathrm{p}$-value is smaller.

\begin{tabular}{cccccccc}
\hline No. & Biological Pathway Name & Affected Genes No. & $\downarrow$ Gene No. & $\uparrow$ Gene No. & FDR & p-value & Z score \\
\hline 1 & TNF signaling pathway (KEGG) & 107 & 68 & 39 & 0.019481 & 0.000999 & -4.7227 \\
2 & NFkB signaling pathway (KEGG) & 88 & 50 & 38 & 0.13247 & 0.016983 & -2.2215 \\
3 & NOD-like receptor signaling pathway (KEGG) & 168 & 99 & 69 & 0.019481 & 0.000999 & -4.4693 \\
4 & Yersinia infection (KEGG) & 114 & 68 & 46 & 0.025974 & 0.001998 & -3.2429 \\
5 & Apoptosis (KEGG) & 85 & 53 & 32 & 0.87662 & 0.008991 & -2.5587 \\
6 & BASE excision repair (KEGG) & 32 & 13 & 19 & 0.54545 & 0.027972 & 1.8866 \\
7 & Keratinization (Wiki pathways) & 137 & 48 & 89 & 0.038961 & 0.000999 & 4.936 \\
8 & Sphingolipid metabolism (KEGG) & 35 & 21 & 14 & 0.23377 & 0.041958 & -1.8128 \\
\hline
\end{tabular}

Table 2. The calculated $\mathrm{Z}$ score values of the expression levels of seven skin relevant biological pathways, affected following skin exposure to PRE and RE.

\begin{tabular}{cccc}
\hline No. & Skin Related Biological Pathway Name & PRE & RE \\
\hline 1 & TNF signaling pathway (KEGG) & -4.7227 & -1.1097 \\
2 & NFKB signaling pathway (KEGG) & -2.2215 & -0.46727 \\
3 & NOD-like receptor signaling pathway (KEGG) & -4.4693 & 0.35018 \\
4 & Yersinia infection (KEGG) & -3.2429 & 1.5054 \\
5 & Apoptosis (KEGG) & -2.5587 & 1.0758 \\
6 & BASE excision repair (KEGG) & 1.8866 & -0.28178 \\
7 & Sphingolipid metabolism (KEGG) & -1.8128 & -0.6417 \\
\hline
\end{tabular}

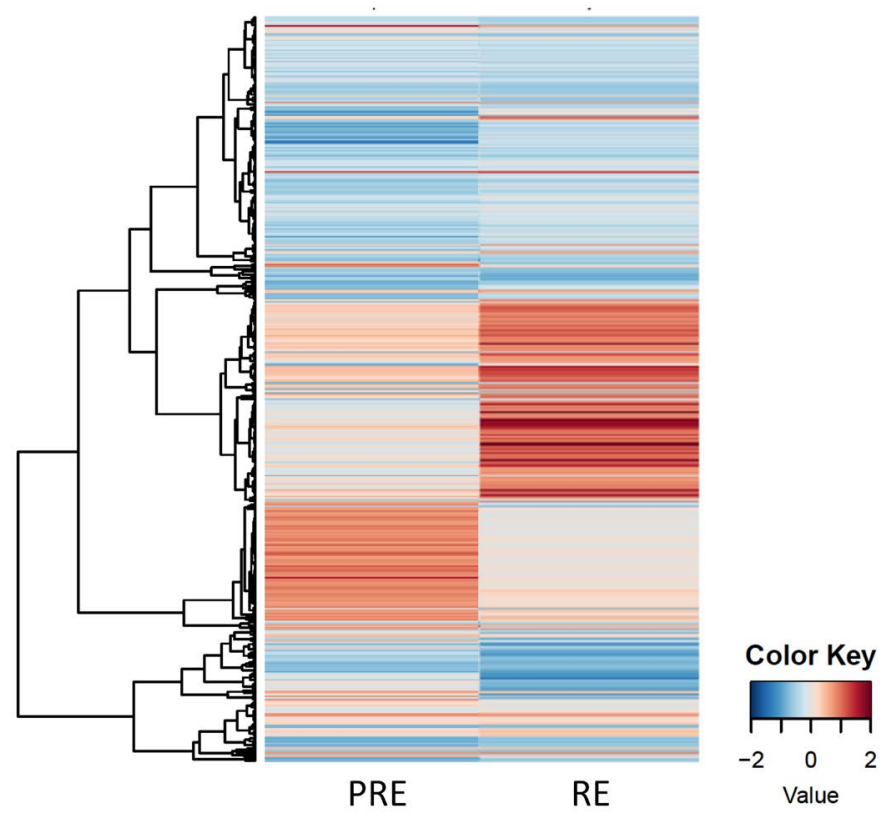

Figure 2. The heatmap of gene expression levels of full-thickness reconstructed human skin, following its exposure to PRE, vs RE treatments. Genes are grouped according to skin relevant biological pathways. The blue shades relate to down regulated genes while red shades represent up regulated genes. 


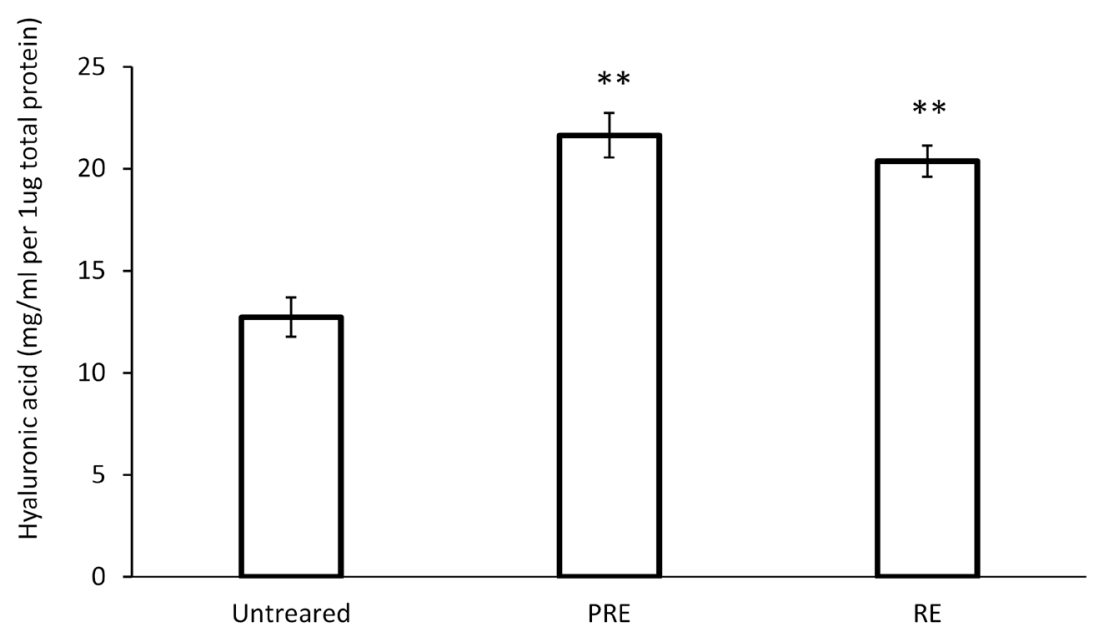

Figure 3. Hyaluronic Acid (HA) levels on fibroblasts dermal cells following their exposure to PRE and $1 \mu \mathrm{M}$ RE. HA levels were quantified in dermal fibroblast cells following cell exposure to the tested materials and incubated at $37^{\circ} \mathrm{C}, 5 \% \mathrm{CO}_{2}$ for $48 \mathrm{~h}$. Untreated fibroblasts cells serve as a control. The values represent the mean $\pm \mathrm{SE}$. " $* *$ " symbolizes significance of $\mathrm{p}<0.001$.
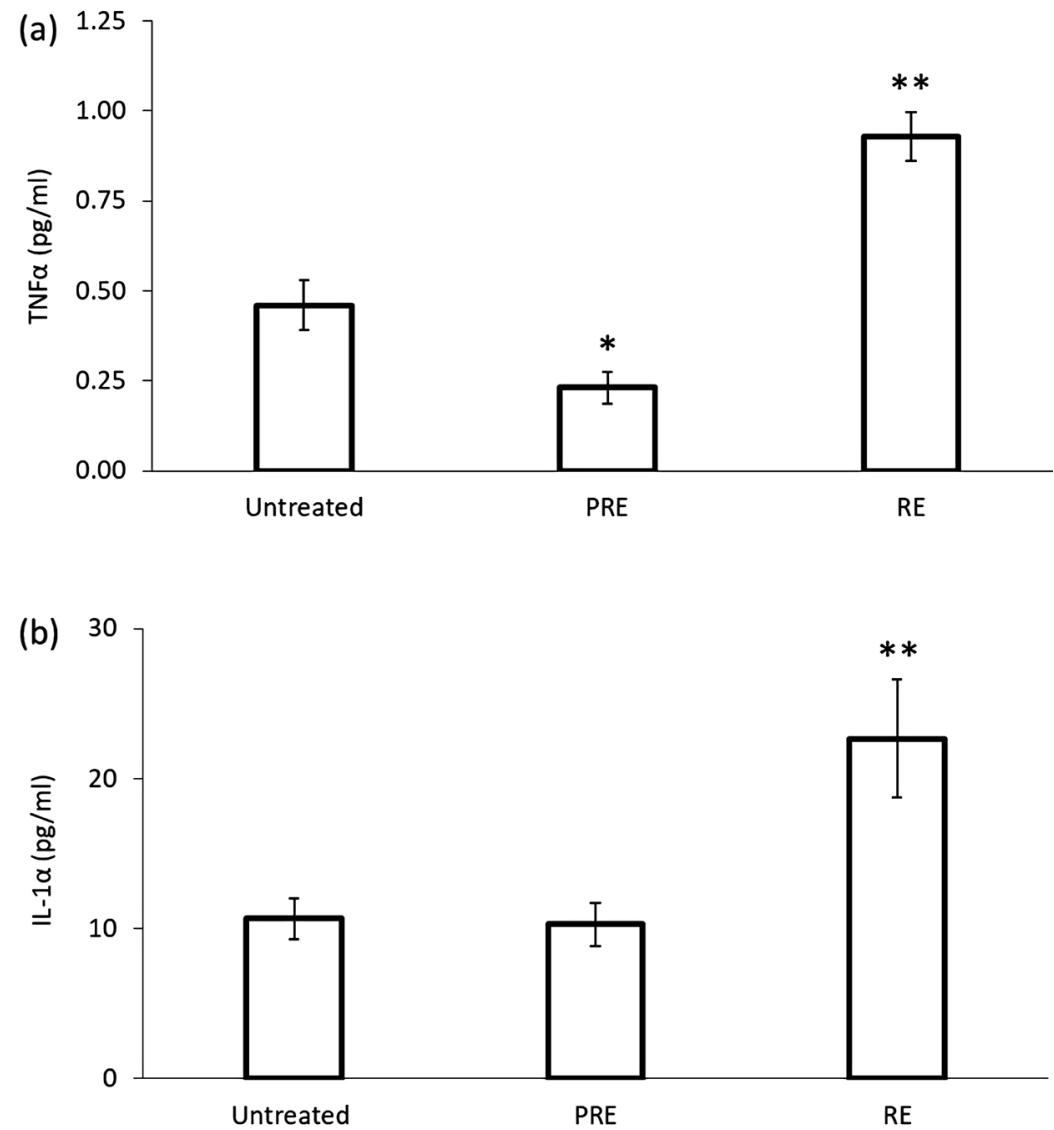

Figure 4. HSOC skin pieces after their exposure to test materials. HSOC skin pieces were topically applied with PRE or 1\% (w/w) RE and incubated for $48 \mathrm{~h}$. After $48 \mathrm{~h}$ the culture media were collected and the cytokines level of TNF $\alpha$ (a) and IL-1 $\alpha$ (b) were determined via ELISA assay. The values represent the mean \pm SE. “*” symbolizes significance of $p<$ 0.05 , while "*** symbolizes significance of $\mathrm{p}<0.001$. 

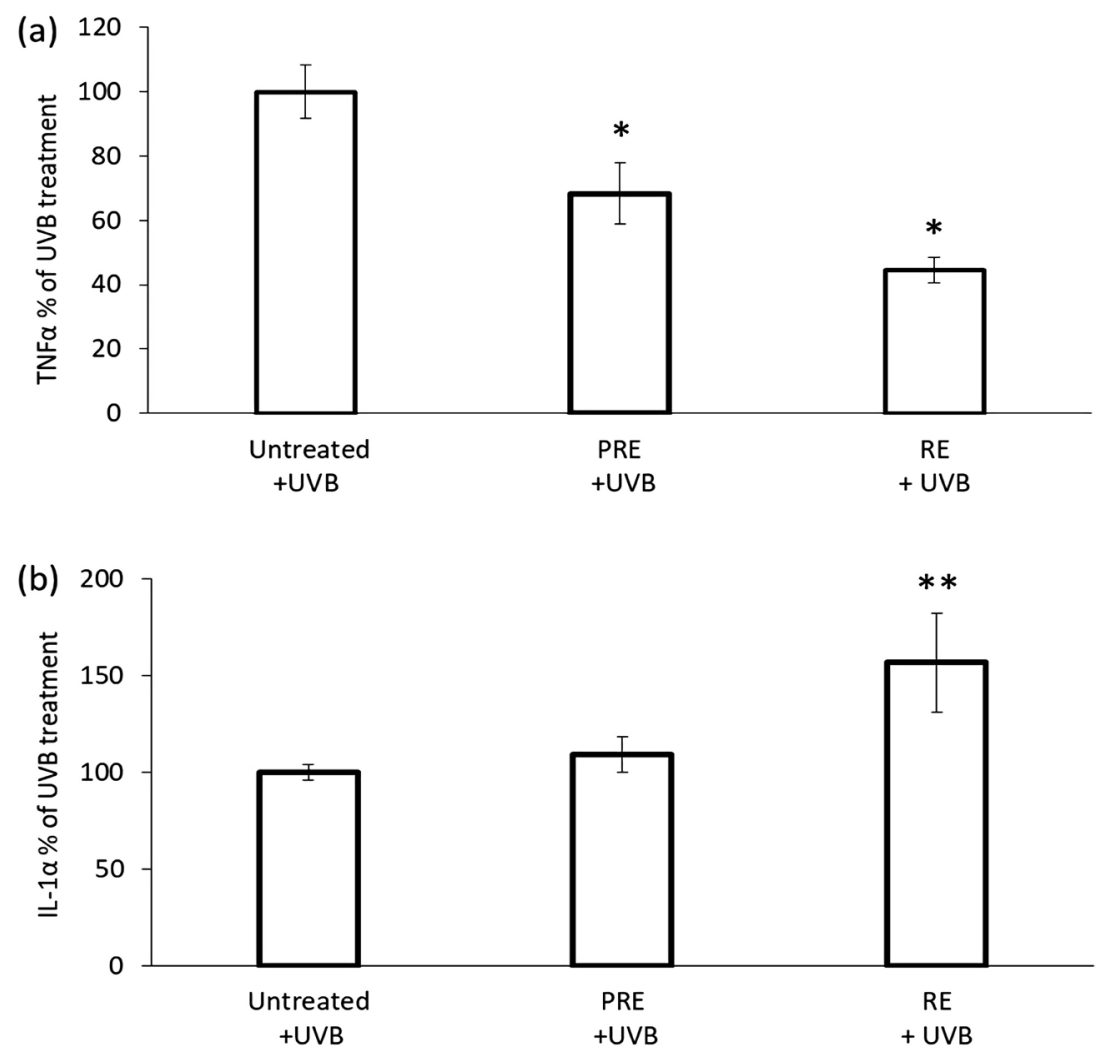

Figure 5. HSOC after test materials' double applications and skin exposure to UVB irradiation as described in the Methods part. HSOC ex-vivo skin pieces were topically applied for $24 \mathrm{~h}$ with test material, then irradiated with $200 \mathrm{~mJ} / \mathrm{cm}^{2}$ UVB lamp. After UVB radiation, the HSOC pieces were re-treated with the test material and incubated for additional $24 \mathrm{~h}$. After $48 \mathrm{~h}$ the culture media were collected and the cytokines level of TNF $a$ (a) and IL-1 $\alpha$ (b) were determined via ELISA assay. The values represent the mean \pm SE. “*” symbolizes significance of $p<0.05$, while “**» symbolizes significance of $p<0.001$.

The results show significant IL- $1 \alpha$ elevation of $57 \%$ and inhibition of TNF $\alpha$ level by $55 \%$ following RE treatment, while PRE treatment was not affecting the levels of IL- $1 \alpha$ and significantly decreased TNF $\alpha$ levels by $32 \%$.

\section{Discussion}

RE involvement in anti-aging skin processes is well established [1] [2] [3]. Due to its positive influence on skin ultrastructure [8], RE is formulated in many products, promising to see and feel the difference in skin appearance within a short term. However, the debate about RE safety reduces its prospective despite its respected value [9]. Legal acts avoid its free usage in global cosmetic preparations. The approved level of RE is restricted within the European Commission's countries to maximum concentration of $0.3 \%$ for hand and facial creams and only up to $0.05 \%$ on body lotions [3]. Health authorities in Canada had lately issued a warning, notice that a change of the legal status of RE is currently considered, aiming to reduce the permitted concentration of RE for certain types of cosmetic products, such as body lotions, face products and hand creams, because its skin 
exposure level may exceed the recommended Tolerable Upper Intake Levels [28]. In The US, retinol can be freely used in cosmetic products with no legal limit however, formulating retinol to treat acne ${ }^{7}$, Hyperpigmentation and wrinkles ${ }^{6}$ might trigger FDA to take enforcement action against drug-like claims. Therefore, when marketing a RE product as a cosmetic item, companies must be careful before making strong claims about a product activity, and its safety should be well established [10] [29]. In some places, manufacturers are guided to add warnings, to avoid RE application by pregnant women because of retinol related teratogenic risks [30]. During the last decade, the influence of NGOs (Non-governmental organizations) on the public awareness has dramatically grown and their today's marketing power is huge. In its "skin-deep" score, "Environmental Working Group" (EWG), a well-recognized NGO, evaluates RE as unsafe [31] and many concerns are raised, including its deep absorbance into the skin [32], a production of excess reactive oxygen species, that might interfere with cellular signaling, cause mutations and lead to cell death, as well as its human reproductive toxic effect [33]. "Skin-deep" cites animal studies that show tumor formation at very low RE dosages and in-vitro tests on mammalian cells show positive mutation results [31] [34]. Consequently, RE fragile safety profile is currently under concerns by both regulation systems and the public awareness. This evaluation energizes the drive to find active ingredients, that would deliver the desired RE skin benefits, with a healthier skin safety profile. RE is naturally created from precursors within our skin, and this safe supply is controlled and catalyzed by enzymatic activity [35]. Offering the possibility to incorporate RE precursors to skin, enabling a controlled self-supply of "natural-to-skin" RE source, is therefore logically concluded. PRE was designed as a complex that combines two important RE precursors, i.e. $\beta$-Carotene and Niacinamide, together with two classical Dead Sea "local" actives, i.e. its mineral saturated water (Osmoter ${ }^{\mathrm{TM}}$ ) and its exceptional alga ( $D u$ naliella Salina). The selected four actives are mixed together to form PRE, a patented active complex, combined of $\beta$-Carotene, Niacinamide, Dunaliella Salina and Dead Sea water (US Pro. Patent App. No. 62/986,698). The RE skin benefit delivered by PRE application, as well as its skin safety, were studied and the results are described in this work. The expression level of typical skin biomarkers was measured using various skin models: In vitro Human dermal fibroblasts cell culture, reconstructed 3D skin equivalent and ex-vivo human skin organ culture. HA is used as a bio marker to represent one of RE described skin benefits, and its expressed level on skin was measured. HA is a polysaccharide that enables the maintenance of essential skin hydration. HA levels in skin decline with age, resulting a decrease in skin moisture, which contributes to loss of firmness and wrinkles formation [36]. RE application is known to encourage HA formation as detected in skin [27] [37]. In this study, HA levels were tested on fibroblasts cells, reported for HA production within the dermis [38]. The study results, presented in Figure 3, support a claim of retinol-like skin benefit. Following the exposure of fibroblasts to PRE, the natural synthesis of HA is significantly elevated in both 
RE and PRE preparations, while PRE exhibits 9\% higher elevation, when compared to RE application. Human skin organ culture (HSOC) was selected as a skin model to compare the effect of PRE vs RE in terms of safety. This ex-vivo model enables to measure the expression of molecules reflecting irritation and inflammation, following topical application of RE and PRE, and the similarity to real product's application on skin is well established. As a preliminary step of safety evaluation, tests for MTT, represent skin viability, were performed. HSOC skin pieces were exposed to PRE and RE and MTT results were measured [25]. No disruption of skin viability was concluded following the application of both RE and PRE (unpublished data). For assessing skin safety, the cytokine biomarkers, TNF $\alpha$ and IL- $1 \alpha$, were chosen and their expression was measured on HSOC. $\mathrm{TNF} \alpha$ is a common inflammation biomarker [39] and IL- $1 \alpha$ is a common irritation bio marker [40]. RE application was compared to PRE and the results, presented in Figure 4, help to evaluate PRE skin safety benefit $v s$ RE. Indication for inflammation and irritation, as shown by the expression of cytokine bio-markers, was observed following RE application, as expected, since skin irritation, and upregulation of the expression of IL- $1 \alpha$ molecule, were reported as side effects of RE topical application [41] [42]. Unlike RE application that results in a significant TNF $\alpha$ and IL- $1 \alpha$ elevation, skin treatment with PRE significantly reduces the levels of expressed TNF $\alpha$ and the level of IL- $1 \alpha$ is not affected. These results may establish a claim for skin safety advantage of PRE when compared to RE.

HSOC skin model was further utilized to investigate how topical application of PRE and RE may influence skin capability to protect itself from external imposed stress of UV radiation. The expression of the two cytokine biomarkers, TNF $\alpha$ and IL- $1 \alpha$, were tested on UVB irradiated HSOC. This model imitates skin following sun exposure and allows to predict PRE safety during external stress in general, and sun-exposed skin in particular. The results, presented in Figure 5, show significant IL- $1 \alpha$ elevation and inhibition of TNF $\alpha$ level, following RE treatment, while PRE treatment was not affecting the levels of IL- $1 \alpha$ and had led to a significant decrease in the expressed level of TNF $\alpha$. These results, together with HA reported observations, suggest that not only PRE delivers retinol-like skin beneficial effect, but it performs a better skin safe profile, as expressed by the attenuation of irritation and inflammation related biomarkers.

In order to better understand the biological mechanisms beyond skin effect, a full genome micro array was performed and the gene expression of skin, before and after the application of tested materials, was assessed. The resulted heatmap, presented in Figure 2, shows the significantly affected genes and biological pathways. Different patterns of pRETINOL and RE are presented, yet there are two areas of similarity of up-regulated genes in the middle of the graph, and down regulated genes in the heatmap top, i.e. some gene upregulation and downregulation are common among the two tested samples. Various pathways, that are relevant to skin biology, seems to be affected by PRE application. The analysis of the affected biological pathways, presented in Table 1 and Table 2, shows that 
PRE treatment reduces the following inflammation-related pathway: TNF signaling, nuclear factor-kappa $\mathrm{B}(\mathrm{NF} \mathrm{B})$ signaling, NOD-like receptor signaling (NLRs) and Yersinia infection, as well as apoptosis. TNF is the mediator of many cytokines, activating the immune system. Being an endogenous pyrogenic agent, TNF is able to induce fever and lead to damage such as inflammation and apoptotic cell death [43]. NFKB activates a family of transcription factors that regulate the transcription of genes that are involved in immunity system [44]. NLRs and Yersinia infections are inflammasome-related pathways. Inflammasomes are cytosolic multi-protein oligomers, participating in the innate immune system, accountable for inflammatory responses activation and play an important role as a first line defense on the epithelial barrier. Activation and assembly of the inflammasome promotes proteolytic cleavage and secretion of pro-inflammatory cytokines, interleukin $1 \beta$ and interleukin 18 [45]. Recent studies had revealed a possible link between the formation of inflammasome structures and skin pathologies including acne, rosacea, atopic dermatitis, psoriasis, and skin inflammaging [46] [47]. Since apoptotic induction is related to the described above inflammatory pathways and because PRE treatment inhibits the inflammatory pathways, it also attenuates the apoptosis pathway, as expected. Unlike PRE, topical application of $\mathrm{RE}$ does not change the inflammatory and apoptosis pathways in skin, but leads to up-regulation of Yesrsinia-related to inflammasome pathway, raising RE safety issue in the level of gene expression and activated biological pathways. Analyzing the results, relating to the biological pathways that regulate NFkB, TNF and apoptosis, may indicate a direction of PRE involvement as skin biological calming and stabilizing agent, working via the expression decline of its inflammation and apoptosis related pathways. The described attenuating effect, following PRE application, is emphasized when results are put in contrast to RE affected pathways, as indicated by a comparison of calculated $\mathrm{Z}$ scores for expressed pathways, described in Table 2. Additional key biological pathway, found to be affected by PRE treatment, is BASE excision repair (BER), which was up-regulated. BER is involved in removing damaged bases of DNA, that could otherwise lead to mispairing mutations or to a breaks in DNA strand during its replication [48]. Interestingly PRE, which induces an upregulation of this pathway may assist to maintain more stable and safer cell replication process. Gene expression results shed a new light on a possible involvement of PRE in terms of inflammatory attenuation and efficient cell proliferation process, hence, contributing to maintain more stable skin functionality.

\section{Conclusion}

The benefit of formulation RE precursors in cosmetic preparations, as a safe source of RE supply to skin, proposed by PRE patented complex, is partially supported. The presented results prove that PRE delivers some beneficial skin bio-activities, which are similar to RE contribution, while revealing safer characteristics to skin. Further researches are needed in order to expose the mechanism beyond the reported skin benefits of PRE application. 


\section{Acknowledgements}

The authors thank Mr. Robert Holtz of BioInnovation Laboratories Inc., and Mr. Xinmin Zhang of BioinfoRx for microarray work and analysis, and Dead Sea Laboratories formulation team for tested material lab preparations.

\section{Conflicts of Interest}

The authors declare no conflicts of interest regarding the publication of this paper.

\section{References}

[1] Al Tanoury, Z., Piskunov, A. and Rochette-Egly, C. (2013) Vitamin A and Retinoid Signaling: Genomic and Nongenomic Effects Thematic Review Series: Fat-Soluble Vitamins: Vitamin A. Journal of Lipid Research, 54, 1761-1775. https://doi.org/10.1194/jlr.R030833

[2] O’Byrne, S.M. and Blaner, W.S. (2013) Retinol and Retinyl Esters: Biochemistry and Physiology Thematic Review Series: Fat-Soluble Vitamins: Vitamin A. Journal of Lipid Research, 54, 1731-1743. https://doi.org/10.1194/jlr.R037648

[3] Bernauer, U., et al. (2016) Scientific Committee on Consumer Safety SCCS Opinion on Vitamin A (Retinol, Retinyl Acetate, Retinyl Palmitate).

[4] Mark, M., Ghyselinck, N.B. and Chambon, P. (2006) Function of Retinoid Nuclear Receptors: Lessons from Genetic and Pharmacological Dissections of the Retinoic Acid Signaling Pathway during Mouse Embryogenesis. Annual Review of Pharmacology and Toxicology, 46, 451-480. https://doi.org/10.1146/annurev.pharmtox.46.120604.141156

[5] Zasada, M. and Budzisz, E. (2020) Randomized Parallel Control Trial Checking the Efficacy and Impact of Two Concentrations of Retinol in the Original Formula on the Aging Skin Condition: Pilot Study. Journal of Cosmetic Dermatology, 19, 437-443. https://doi.org/10.1111/jocd.13040

[6] Draelos, Z.D. (2005) Novel Approach to the Treatment of Hyperpigmented Photodamaged Skin: 4\% Hydroquinone/0.3\% Retinol versus Tretinoin 0.05\% Emollient Cream. Dermatologic Surgery, 31, 799-805. https://doi.org/10.1111/j.1524-4725.2005.31723

[7] Thielitz, A. and Gollnick, H. (2008) Topical Retinoids in Acne Vulgaris. American Journal of Clinical Dermatology, 9, 369-381. https://doi.org/10.2165/0128071-200809060-00003

[8] Zasada, M. and Budzisz, E. (2019) Retinoids: Active Molecules Influencing Skin Structure Formation in Cosmetic and Dermatological Treatments. Advances in Dermatology and Allergologyl Postepy Dermatologii i Alergologii, 36, 392. https://doi.org/10.5114/ada.2019.87443

[9] Kang, S., et al. (1995) Application of Retinol to Human Skin in Vivo Induces Epidermal Hyperplasia and Cellular Retinoid Binding Proteins Characteristic of Retinoic Acid But without Measurable Retinoic Acid Levels or Irritation. Journal of Investigative Dermatology, 105, 549-556. https://doi.org/10.1111/1523-1747.ep12323445

[10] Ries, G. and Hess, R. (1999) Retinol: Safety Considerations for Its Use in Cosmetic Products. Journal of Toxicology: Cutaneous and Ocular Toxicology, 18, 169-185. https://doi.org/10.3109/15569529909044238 
[11] Lee, S.J., et al. (2005) Cosmetic Material Containing Triple-Encapsulated Retinol. Google Patents.

[12] Gonçalves, A., Estevinho, B.N. and Rocha, F. (2016) Microencapsulation of Vitamin A: A Review. Trends in Food Science \& Technology, 51, 76-87. https://doi.org/10.1016/j.tifs.2016.03.001

[13] Antille, C., et al. (2004) Topical $\beta$-Carotene Is Converted to Retinyl Esters in Human Skin ex Vivo and Mouse Skin in Vivo. Experimental Dermatology, 13, 558-561. https://doi.org/10.1111/j.0906-6705.2004.00194.x

[14] Andersson, E., Vahlquist, A. and Rosdahl, I. (2001) $\beta$-Carotene Uptake and Bioconversion to Retinol Differ between Human Melanocytes and Keratinocytes. $\mathrm{Nu}$ trition and Cancer, 39, 300-306. https://doi.org/10.1207/S15327914nc392_21

[15] Oblong, J.E. (2017) Retinyl Propionate and Related Retinoids. In: Textbook of Cosmetic Dermatology, CRC Press, Boca Raton, 83-87.

[16] Hu, K.-Q., et al. (2006) The Biochemical Characterization of Ferret Carotene-9', 10'-Monooxygenase Catalyzing Cleavage of Carotenoids in Vitro and in Vivo. Journal of Biological Chemistry, 281, 19327-19338. https://doi.org/10.1074/jbc.M512095200

[17] Portugal-Cohen, M., et al. (2019) Dead Sea Minerals: New Findings on Skin and the Biology Beyond. Experimental Dermatology, 28, 585-592. https://doi.org/10.1111/exd.13918

[18] Harari, M. (2012) Beauty Is Not Only Skin Deep: The Dead Sea Features and Cosmetics. Anales de Hidrología Médica, 5, 75-88.

[19] Oren, A. (2005) A Hundred Years of Dunaliella Research: 1905-2005. Saline Systems, 1, 2. https://doi.org/10.1186/1746-1448-1-2

[20] Emeish, S. (2012) Production of Natural $\beta$-Carotene from Dunaliella Living in the Dead Sea. Jordan Journal of Earth and Environmental Sciences, 4, 23-27.

[21] Herrmann, H., et al. (1996) Effects of UV Radiation on Photosynthesis of Phytoplankton Exposed to Solar Simulator Light. Journal of Photochemistry and Photobiology B: Biology, 34, 21-28. https://doi.org/10.1016/1011-1344(95)07245-4

[22] Portugal-Cohen, M., et al. (2011) Skin Organ Culture as a Model to Study Oxidative Stress, Inflammation and Structural Alterations Associated with UVB-Induced Photodamage. Experimental Dermatology, 20, 749-755. https://doi.org/10.1111/j.1600-0625.2011.01317.x

[23] Limat, A., et al. (1989) Post-Mitotic Human Dermal Fibroblasts Efficiently Support the Growth of Human Follicular Keratinocytes. Journal of Investigative Dermatology, 92, 758-762. https://doi.org/10.1016/0022-202X(89)90196-6

[24] Walker, J.M. (2009) The Bicinchoninic Acid (BCA) Assay for Protein Quantitation. In: The Protein Protocols Handbook, Springer, Berlin, 11-15. https://doi.org/10.1007/978-1-59745-198-7_3

[25] Portugal-Cohen, M., et al. (2018) Apple of Sodom (Calatropis procera) Callus Extract, a Novel Skincare Active and Its Biological Activity in Skin Models When Combined with Dead Sea Water. Journal of Cosmetics, Dermatological Sciences and Applications, 8, 73-91. https://doi.org/10.4236/jcdsa.2018.82010

[26] Desktop, G. v3.0 Broad Institute. Massachusetts Institute of Technology, Cambridge. http://software.broadinstitute.org/gsea/index.jsp

[27] Li, W.-H., et al. (2017) Topical Stabilized Retinol Treatment Induces the Expression of HAS Genes and HA Production in Human Skin in Vitro and in Vivo. Archives of Dermatological Research, 309, 275-283. https://doi.org/10.1007/s00403-017-1723-6 
[28] Branch, C.S. (2004) Cosmetic Ingredient Hotlist-Health Canada.

[29] Yourick, J.J., Jung, C.T. and Bronaugh, R.L. (2008) In Vitro and in Vivo Percutaneous Absorption of Retinol from Cosmetic Formulations: Significance of the Skin Reservoir and Prediction of Systemic Absorption. Toxicology and Applied Pharmacology, 231, 117-121. https://doi.org/10.1016/j.taap.2008.04.006

[30] Tzimas, G. and Nau, H. (2001) The Role of Metabolism and Toxicokinetics in Retinoid Teratogenesis. Current Pharmaceutical Design, 7, 803.

https://doi.org/10.2174/1381612013397708

[31] EWG’s Skin Deep. https://www.ewg.org/skindeep/ingredients/706889-retinol_(vitamin_a)

[32] Antille, C., et al. (2004) Penetration and Metabolism of Topical Retinoids in ex Vivo Organ-Cultured Full-Thickness Human Skin Explants. Skin Pharmacology and Physiology, 17, 124-128. https://doi.org/10.1159/000077238

[33] Gelain, D.P. and Moreira, J.C.F. (2008) Evidence of Increased Reactive Species Formation by Retinol, But Not Retinoic Acid, in PC12 Cells. Toxicology in Vitro, 22, 553-558. https://doi.org/10.1016/j.tiv.2007.11.007

[34] Vooijs, M., et al. (2002) Tumor Formation in Mice with Somatic Inactivation of the Retinoblastoma Gene in Interphotoreceptor Retinol Binding Protein-Expressing Cells. Oncogene, 21, 4635-4645. https://doi.org/10.1038/sj.onc.1205575

[35] Roos, T.C., et al. (1998) Retinoid Metabolism in the Skin. Pharmacological Reviews, 50, 315-333.

[36] Longas, M.O., Russell, C.S. and He, X.-Y. (1987) Evidence for Structural Changes in Dermatan Sulfate and Hyaluronic Acid with Aging. Carbohydrate Research, 159, 127-136. https://doi.org/10.1016/S0008-6215(00)90010-7

[37] Tammi, R. and Tammi, M. (1986) Influence of Retinoic Acid on the Ultrastructure and Hyaluronic Acid Synthesis of Adult Human Epidermis in Whole Skin Organ Culture. Journal of Cellular Physiology, 126, 389-398. https://doi.org/10.1002/jcp.1041260309

[38] Harper, R.A. (1988) Specificity in the Synergism between Retinoic Acid and EGF on the Growth of Adult Human Skin Fibroblasts. Experimental Cell Research, 178, 254-263. https://doi.org/10.1016/0014-4827(88)90396-5

[39] Grellner, W., Georg, T. and Wilske, J. (2000) Quantitative Analysis of Proinflammatory Cytokines (IL-1 $\beta$, IL-6, TNF- $\alpha$ ) in Human Skin Wounds. Forensic Science International, 113, 251-264. https://doi.org/10.1016/S0379-0738(00)00218-8

[40] Martinez, V., et al. (2006) Evaluation of Eye and Skin Irritation of Arginine-Derivative Surfactants Using Different in Vitro Endpoints as Alternatives to the in Vivo Assays. Toxicology Letters, 164, 259-267. https://doi.org/10.1016/j.toxlet.2006.01.005

[41] Trechsel, U., Evêquoz, V. and Fleisch, H. (1985) Stimulation of Interleukin 1 and 3 Production by Retinoic Acid in Vitro. Biochemical Journal, 230, 339-344. https://doi.org/10.1042/bj2300339

[42] Hashimoto, S., et al. (1998) Retinoic Acid Differentially Regulates Interleukin-1 $\beta$ and Interleukin-1 Receptor Antagonist Production by Human Alveolar Macrophages. Leukemia Research, 22, 1057-1061. https://doi.org/10.1016/S0145-2126(98)00119-2

[43] Yeretssian, G., Labbé, K. and Saleh, M. (2008) Molecular Regulation of Inflammation and Cell Death. Cytokine, 43, 380-390. https://doi.org/10.1016/j.cyto.2008.07.015 
[44] Vallabhapurapu, S. and Karin, M. (2009) Regulation and Function of NF- $\kappa$ B Transcription Factors in the Immune System. Annual Review of Immunology, 27, 693-733. https://doi.org/10.1146/annurev.immunol.021908.132641

[45] Watanabe, H., et al. (2007) Activation of the IL-1 $\beta$-Processing Inflammasome Is Involved in Contact Hypersensitivity. Journal of Investigative Dermatology, 127, 1956-1963. https://doi.org/10.1038/sj.jid.5700819

[46] Sá, D.C.D. and Festa Neto, C. (2016) Inflammasomes and Dermatology. Anais Brasileiros de Dermatologia, 91, 566-578. https://doi.org/10.1590/abd1806-4841.20165577

[47] Menu, P. and Vince, J. (2011) The NLRP3 Inflammasome in Health and Disease: The Good, the Bad and the Ugly. Clinical \& Experimental Immunology, 166, 1-15. https://doi.org/10.1111/j.1365-2249.2011.04440.x

[48] Melis, J.P., van Steeg, H. and Luijten, M. (2013) Oxidative DNA Damage and Nucleotide Excision Repair. Antioxidants \& Redox Signaling, 18, 2409-2419. https://doi.org/10.1089/ars.2012.5036 